Classification

Physics Abstracts

$61.30-61.40 \mathrm{~K}$

\title{
Side chain mesomorphic polymers : studies of labelled backbones by neutron scattering
}

\author{
P. Keller, B. Carvalho (*), J. P. Cotton, M. Lambert, F. Moussa and G. Pépy \\ Laboratoire Léon Brillouin (**), CEN-Saclay, 91191 Gif-sur-Yvette Cedex, France
}

(Reçu le 8 août 1985, accepté le 27 septembre 1985)

\begin{abstract}
Résumé. - Des polymères mésomorphes en peigne sont étudiés dans les phases isotrope, nématique et smectique. Les échantillons sont formés de mélanges de polyméthacrylates cristaux liquides dont les squelettes sont deutériés ou hydrogénés. Ils sont étudiés par diffraction et diffusion de neutrons aux petits angles. Les résultats montrent en particulier que dans la phase smectique alignée : i) le squelette présente une forte anisotropie, il est confiné dans une couche smectique.ii) Le marquage du squelette fait apparaître un ordre local en bicouche.
\end{abstract}

Abstract. - Side chain liquid crystal polymers are studied in the isotropic, nematic and smectic
phases. The samples are made from a mixture of liquid crystal polymethacrylate with deuterated or
protonated backbones. The samples are studied with neutron diffraction and small angle neutron
scattering. The results show in particular that in the aligned smectic phase the backbone is highly
anisotropic and confined within a smectic layer. The backbone labelling shows local bilayered order.

The liquid crystalline polymers studied here are comb like polymers, i.e. each monomer of the backbone is connected with a mesogenic molecule. The bulk properties of these polymers show the existence of phases (isotropic, nematic and smectic) characteristic of liquid crystals. Thus the mesogenic molecules impose their order on the system and the main problem [1] is how this order influences the conformation and the organization of the chains.

From X-ray measurements [2], it is assumed that in the aligned smectic phase the backbone is strongly confined between the mesogenic layers. Such an assumption has to be confirmed by the determination of the anisotropy of a chain conformation. This can be achieved with a labelling method.

Deuteration of the backbone associated with neutron diffraction (ND) and small angle neutron scattering (SANS) is the main tool for resolving such problems because this labelling method allows to determine the system organization (from ND [3]) and the chain conformation (from SANS [4]). A first experiment [5] performed with SANS has shown an anisotropy of about $25 \%$ of the radius of gyration in the nematic phase; experimental difficulties prevented the authors from performing similar experiments in the aligned smectic phase.

$\left(^{*}\right)$ Permanent address : Center for Polymer Studies, Boston University, MA 02215, USA.

$(* *)$ Laboratoire Commun CEA-CNRS. 
The results discussed here are obtained with four samples, each with a different value of the concentration $\phi(\phi=0 ; 0.25 ; 0.50$ and 1$)$ of chains with deuterated backbone. It should be noted that between the sample $\phi=0$ and $\phi=1$ the number of protons replaced by deuterium varies only $15 \%$.

The experimental part is discussed in section 1, the results concerning the chain conformation in section 2 and that for the chain organization in section 3.

\section{Samples and experimental.}

Liquid crystalline polymethacrylate PMH and PMD were obtained by free radical polymerization of corresponding methacrylate monomers in solution. The synthesis of these monomers was adapted from published method [6-8]. The molecular weight is about $3 \times 10^{5}$.

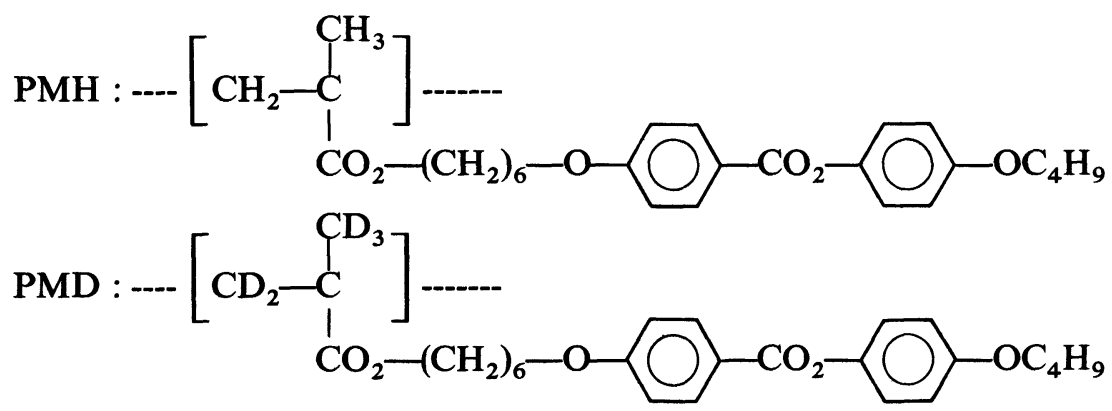

These polymers are similar to those of reference [5] since the backbone and the spacer $\left(\mathrm{CH}_{2}\right)_{6}$ are the same ; they differ only by the terminal part $\left(\mathrm{C}_{4} \mathrm{H}_{9}\right)$ of mesogenic molecule.

The inherent viscosities $\eta$ of PMH and PMD, measured in chloroform $\left(25^{\circ} \mathrm{C}, 5 \times 10^{-3}{\mathrm{~g} . \mathrm{cm}^{-3}}^{-3}\right.$ were found to be of the same order of magnitude (see Table I). This might indicate that the polymers have similar molecular weight distributions. This is confirmed by the similarity of the transition temperatures, listed in table I which were obtained by optical microscopy and DSC experiments.

The neutron experiments were made with the SANS spectrometers PACE and PAXY at the Reactor Orphée (CEN-Saclay).

The radii of gyration were measured with polymers dissolved in deuterated benzene on PACE (isotropic radial multidetector; scattering vector range : $0.007 \leqslant q \leqslant 0.07 \AA^{-1}$ ). The data for one species (PMH or PMD) were obtained from solutions at four polymer concentrations (between 0.005 and $0.03 \mathrm{~g} . \mathrm{cm}^{-3}$ ). The well-known Zimm procedure [9] was used to extrapolate at zero concentration. With these parameter values the inverse scattered intensities plotted as a function of $q^{2}$ show linear dependence. The results in table I show the similarity of species PMH and PMD.

Table I. - Characteristic parameters of polymers with hydrogenous backbone (PMH) or deuterated backbone (PMD). $\eta$ is the intrinsic viscosity and $R_{\mathrm{g}}$ the radius of gyration in $\mathrm{C}_{6} \mathrm{D}_{6}$.

\begin{tabular}{|c|c|c|c|c|c|c|c|c|c|}
\hline Polymer & $\left(\mathrm{cm}^{3} \mathrm{~g}^{-1}\right)$ & \multicolumn{7}{|c|}{$\begin{array}{l}\text { Transition temperature } \\
\qquad\left({ }^{\circ} \mathrm{C}\right)\end{array}$} & $\begin{array}{l}R_{\mathrm{g}} \\
(\AA)\end{array}$ \\
\hline $\begin{array}{l}\text { PMH } \\
\text { PMD }\end{array}$ & $\begin{array}{l}38 \\
44\end{array}$ & 焉 & $\begin{array}{l}40-45 \\
45-50\end{array}$ & 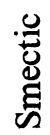 & $\begin{array}{l}110 \\
110\end{array}$ & 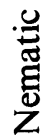 & $\begin{array}{l}115 \\
116\end{array}$ & 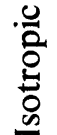 & $\begin{array}{l}166 \pm 10 \\
169 \pm 10\end{array}$ \\
\hline
\end{tabular}


The other results described in the following were obtained with the spectrometer PAXY ( $X Y$ multidetector; cells of $5 \times 5 \mathrm{~mm}^{2}$; at $2 \mathrm{~m}$ of the sample) needed for an anisotropic intensity observation. Two wavelengths $\lambda(\Delta \lambda / \lambda=12 \%)$ were used : $\lambda=15.4 \AA$ for SANS experiments $\left(8 \times 10^{-3} \leqslant q \leqslant 3 \times 10^{-2} \AA^{-1}\right)$ and $\lambda=3.13 \AA$ for ND experiments $\left(0.04<q<0.45 \AA^{-1}\right)$.

The samples (thickness $1 \mathrm{~mm}, 15 \mathrm{~mm}$ of diameter, neutron beam diameter $7 \mathrm{~mm}$ ) were set in an oven (temperature regulation better than $0.2^{\circ} \mathrm{C}$ ). The oven was then placed in a magnetic field of 1.4 tesla in order to obtain aligned nematic and smectic phases.

The liquid crystal phases of the sample were obtained in the following way, the sample was heated into the isotropic phase; then cooled to the nematic phase and aligned in the magnetic field for about two hours. After the sample was measured, it was cooled again to the smectic phase. The degree of alignment was measured from the mosaicity of the single domain (Neutron Diffraction Measurement).

Figure 1 shows a typical pattern obtained with the sample $\phi=0.5$ in the aligned smectic phase. In the centre of the detector one can see the Bragg peak 001 of the smectic phase. Since the Full Width at Half Maximum corresponds to a mosaicity of $5^{\circ}$ the alignment is considered to be good (similar results are obtained with all of the samples). The well observed at the right part of figure 1 is due to the beam catcher. Around this well, figure 1 shows clearly a strong anisotropic forward scattering due to the chain conformation. It is this forward scattering which is studied at $\lambda=$ $15.4 \AA$ by SANS.

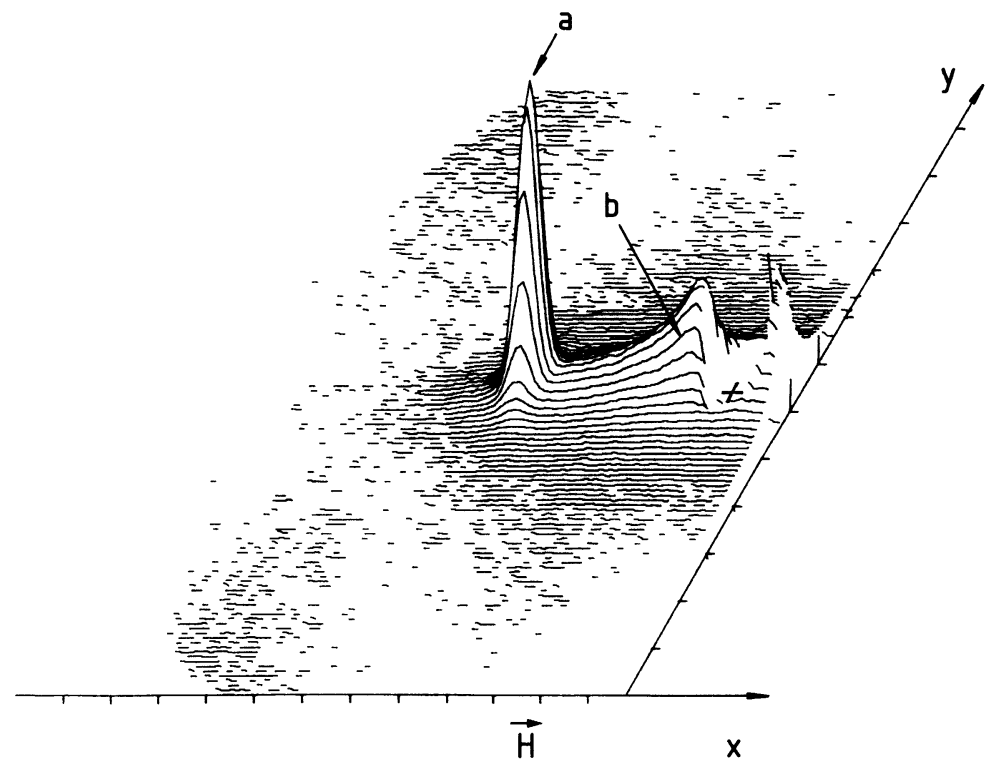

Fig. 1. $-X Y$ spectrum measured at $\lambda=3.13 \AA$ on the aligned smectic phase of the sample with $\phi=0.5$ $(50 \%$ of the backbones are labelled). The cross indicates the position of the direct beam (i.e. the origin of the axis $q_{x}$ and $q_{y}$ ). $H$ is the magnetic field parallel to $x$ axis (a) (001) Bragg peak; (b) anisotropic small angle scattering (see text).

\section{SANS and the chain conformation.}

In this section only the samples $\phi=0.5$ and $\phi=0.25$ are discussed since the others $(\phi=0$ or 1$)$ do not give forward scattering (thus, this scattering corresponds really to the backbone response). 
Let us call $x$ the direction parallel to the magnetic field and $y$ the perpendicular one. The data treatment used in order to obtain characteristic lengths, is obtained from the relation :

$$
I^{-1}\left(q_{i}\right)=I^{-1}(0)\left(1+q_{i}^{2} R_{i}^{2}\right) ; i=x \text { or } y
$$

where $I\left(q_{i}\right)$ is the scattering intensity along the $i$ axis. Equation (1) assumes $[4,5]$ that the molecular weight of PMH and PMD are similar. This last assumption is confirmed $a$ posteriori since the values of $R_{i}$ are $\phi$ independent. $R_{i}$ will be called here the quadratic radius which is related to the radius of gyration $R_{\mathrm{g}}$ in equation (2) :

$$
R_{\mathrm{g}}=R_{i} \sqrt{3}
$$

when the chain has an isotropic conformation. Table II gives the values of $R_{i}$ obtained in the three phases.

Table II. - Values of the quadratic radius (in $\AA$ ) of the backbone for both samples. $x$ and $y$ denote the axes parallel and perpendicular to the magnetic field respectively.

\begin{tabular}{|l|c|c|c|c|c|}
\hline \multirow{4}{*}{$\phi$} & Isotropic & \multicolumn{2}{|c|}{ Nematic } & \multicolumn{2}{c|}{ Smectic } \\
\cline { 2 - 6 } & $R_{x}=R_{y}=\frac{R_{\mathrm{g}}}{\sqrt{3}}$ & $R_{x}$ & $R_{y}$ & $R_{x}$ & $R_{y}$ \\
\hline 0.5 & $61 \pm 5$ & $59 \pm 5$ & $65 \pm 5$ & $22 \pm 3$ & $86 \pm 9$ \\
0.25 & $61 \pm 5$ & & & $21 \pm 3$ & $86 \pm 9$ \\
\hline
\end{tabular}

The data of table II show the following results : in the isotropic phase the value of the radius of gyration $R_{\mathrm{g}}=106 \AA$ is well smaller than that found in benzene $(169 \AA)$. This difference is certainly due to the contrast of mesogenic molecules which is cancelled in the isotropic phase but not in the solvent and probably also to a swelling of the chain in the solvent.

In the nematic phase the data are obtained by regrouping five of the elementary cells of PAXY along $x$ and $y$ axis in order to obtain an intensity with a better statistic. The results analysis yields an anisotropy of $10 \%$ on the $R_{i}$ value. This corresponds to an anisotropy of $20 \%$ on the measured intensity two times weaker than the result first obtained in this phase by Kirste and Ohm [5].

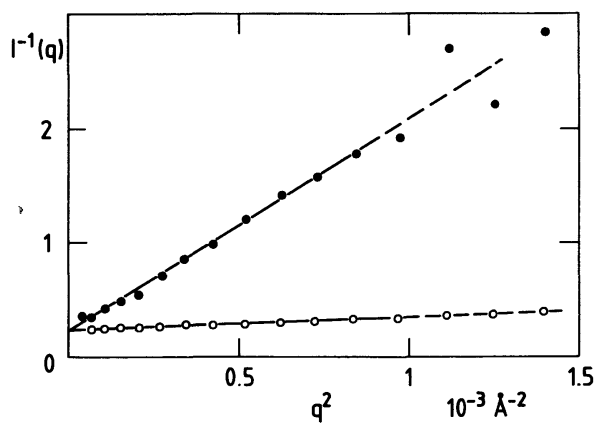

Fig. 2. - The inverse of the small angle scattered intensity versus $q^{2}$. The slopes of both straight lines are $R_{x}^{2}$ and $R_{y}^{2}\left(O: q^{2}=q_{x}^{2} ; \bullet: q^{2}=q_{y}^{2}\right)$. 
Because of the strong anisotropy of the backbone intensity (Fig. 2), in the smectic phase, the data are obtained by regrouping three elementary cells of PAXY only. Figure 2 shows the data of sample $\phi=0.25$ plotted in the representation of equation (1). Assuming that the chain is isotropic in the smectic layers (two dimensional isotropy), the parallel radius of gyration $R_{\|}$and the perpendicular one $R_{\perp}$ have the following values

$$
R_{\|}=R_{x}=21 \AA ; \quad R_{\perp}=R_{y} \sqrt{2}=120 \AA .
$$

\section{Diffraction and chain organization.}

Coherent neutron scattering spectra have been measured at a wave-length of $3.13 \AA$, on aligned samples, with different concentrations $\phi$ of labelled backbone chains.

The spectrum corresponding to the $\phi=1$ sample (Fig. 3) clearly exhibits : a Bragg peak (001) characteristic of a long range layered order (Fig. 3a). It gives a smectic layer thickness of $29.5 \AA$ the same as measured by X-rays [2] — but the second order peak, (002), is not seen with neutrons, while it is well measured with X-rays; ii) two sets of diffuse spots that are symmetric with respect to the $x$ axis, the axis of the magnetic field ; one pair of spots is centred at $q_{x}=0.11 \AA^{-1}$ and $q_{y}= \pm 0.165 \AA^{-1}$ (Fig. 3), the other pair at $q_{x}=0.41 \AA^{-1}$ and $q_{y}= \pm 0.37 \AA^{-1}$, near the edge of the multidetector (Fig. 3d) and then located with a lower precision. These last spots will be referred in the following as large angle diffuse spots.

The fully protonated sample does not yield a so rich spectrum, only the (001) peak is seen.

For intermediate $\phi$ values, the intensity of the (001) Bragg peak decreases with increasing $\phi$ values. The intensity of the diffuse spot at large angles which appear in the $\phi \neq 0$ sample, increases

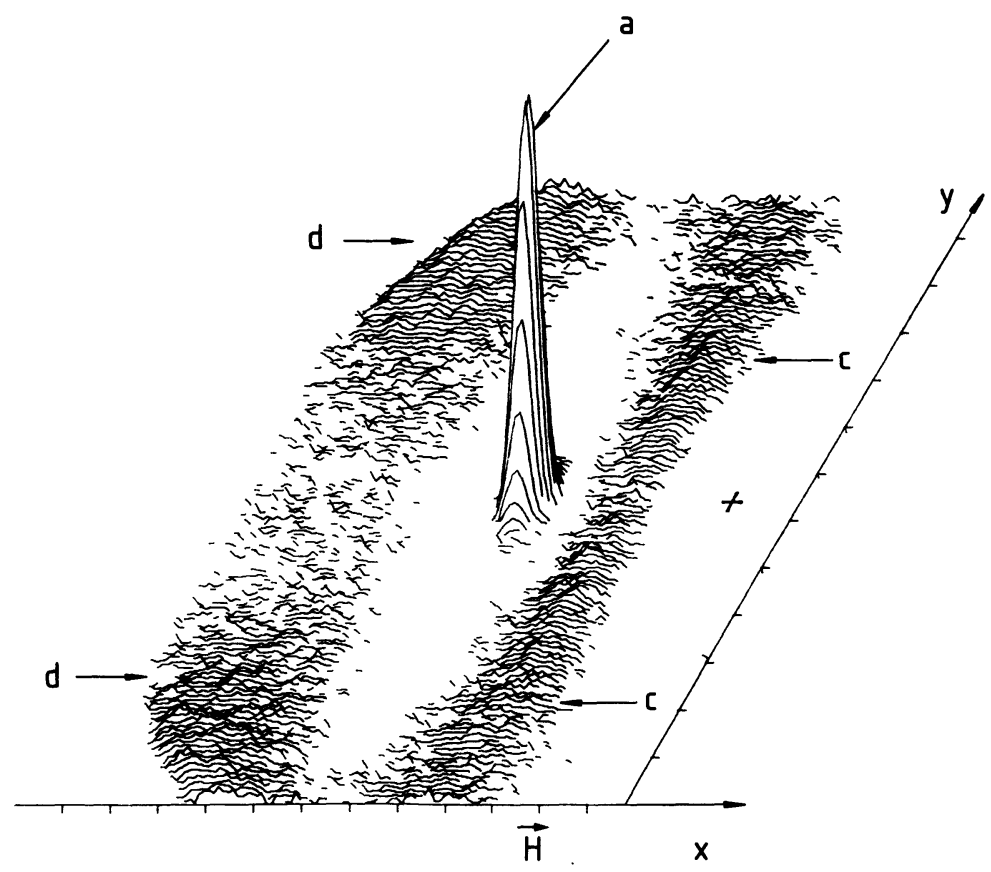

Fig. 3. $-X Y$ spectrum taken in the same conditions as in figure 1 on a sample with fully deuterated backbones : $\phi=1$. (a) (001) Bragg peak; (c) small angle diffuse spots; (d) large angle diffuse spots (see text). One notes the lack of the small angle scattering. 
when $\phi$ goes up to 1 . The smaller angle diffuse spots are visible on the different spectra $(\phi \neq 0)$, for $\phi \neq 1$ they partially merge into the small angle scattering(Fig. 1).

From comparison with X-ray measurements (2), it appears that : i) (001) peak and large angle diffuse spots are well measured by both techniques, ii) the (002) peak, visible with X-rays, is not detectable with neutrons, whatever the sample; iii) and finally the neutron see small angle diffuse spots which are only guessed with X-rays [10].

A qualitative explanation can be given : the intensity of the (001) peak decreases by a factor of 10 when $\phi$ changes its value from zero to one, because the contrast changes with the concentration of labelled backbones.

On the other hand, the small angle diffuse spots correspond to a characteristic distance of $56 \AA$ along the $x$ direction which is approximatively twice the smectic layer thickness $(29.5 \AA)$ and to a pseudoperiod of about $38 \AA$ along the $y$ direction in the smectic layer. The mutual affinity of the terminal part of mesogenic cores $\mathrm{C}_{4} \mathrm{H}_{9}$ may explain this tendancy to make bilayers. This short range order has a coherence length greater than about $50 \AA$ in the direction of the field, and of $10 \AA$ in the perpendicular direction.

\section{Conclusion.}

The labelling of the backbones gives promising results on the anisotropic conformation of the polymer and on its contribution to the structure of the smectic phase. In the parallel $(x)$ direction the size of a polymer $\left(R_{\|}=22 \AA\right)$ is just greater than a length of one mesogenic molecule. In the perpendicular direction this size is six times larger than the size $R_{\|}$. A tentative drawing may be done (Fig. 4) to relate the two sets of results (ND and SANS). It leaves unchanged the position of the 001 Bragg peak. A corresponding model should take into account the influence of the correlation between alignment defects of mesogenic molecules due to the chain connectivity and of the affinity of the terminal parts.

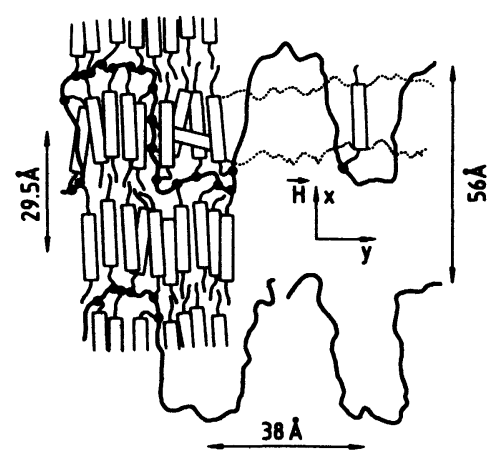

Fig. 4. - Tentative representation of the sample. (For drawing easiness the backbones were restricted to the figure plane.)

To better understand the organization and the conformation of the liquid crystal polymer further experiments are needed; in particular diffraction measurements in samples with new different selective deuteration.

\section{Acknowledgments.}

We wish to thank Dr. B. Farnoux for his invaluable assistance during the measurements on the PAXY spectrometer and Dr. P. Davidson for clarifying discussions. 


\section{References}

[1] Wendorff, J. H., Finkelmann, H., Ringsdorf, H., J. Polym. Sci., Polym. Symp. 63 (1978) 245.

[2] Davidson, P., Keller, P., Levelut, A. M., J. Physique 46 (1985) 939.

Davidson, P., Thèse $3 e$ cycle, Université Paris-Sud (1984).

[3] Bacon, G. E., Neutron Diffraction (Clarendon Press Oxford) 1975.

[4] Cotton, J. P., Decker, D., Benoit, H., Farnoux, B., Higgins, J., Jannink, G., Des Cloizeaux, J., OBER, R., Picot, C., Macromolecules 7 (1974) 863.

[5] KIRSte, R. G., Оhм, H. G., Makromol. Chem., Rapid Commun. 6 (1985) 179.

[6] Portugall, M., Ringsdorf, H., Zentel, R., Makromol. Chem. 183 (1982) 2311.

[7] Klesper, E., Johnsen, A., Granski, W., Wehrli, F. W., Makromol. Chem. 176 (1975) 1071.

[8] Shannon, P. J., Macromolecules 16 (1983) 1677.

[9] Ziмm, B. H., J. Chem. Phys. 16 (1948) 1093.

[10] Davidson, P., Private communication. 